\title{
DESAFÍOS EN LA TRANSFERENCIA DE TECNOLOGÍA A LOS CENTROS EDUCATIVOS
}

\author{
Challenges in the technology transfer to educational \\ centres
}

\section{Des défis dans le transfert de technologie aux centres éducatives}

José PeIrats Chacón* y Ángel SAn MarTín AlOnso**

Universitàt de Valencia. Facultat de Filosofia i Ciències de l'Educació.

Departament de Didàctica i Organització Escolar. Avda. Blasco Ibáñez, 30.

46010 València. Correo-e: *jose.peirats@uv.es, **asanmart@uv.es

Fecha de recepción: marzo de 2012

Fecha de aceptación definitiva: julio de 2012

Biblid [(1130-3743) 24, 2-2012, 215-235]

RESUMEN

Partimos del modelo de transferencia de tecnología para analizar cómo los centros escolares reciben e incorporan a sus actividades los equipamientos tecnológicos. Particularmente analizamos las innovaciones generadas por esos procesos en el plano organizativo de los centros como receptores de la tecnología. Los datos analizados se desagregan de una investigación que actualmente se está realizando en 12 comunidades autónomas sobre el programa Escuela 2.0. En esta fase manejamos estadígrafos descriptivos y referencias de algunas de las entrevistas y de las observaciones de aula realizadas en los centros que participan en el proyecto "centro educativo inteligente». Los primeros análisis nos permiten señalar que en el caso del sistema escolar se sigue un patrón de transferencia muy peculiar: alta dependencia de las instancias políticas, se pone más énfasis en el equipamiento instrumental 
que en el conocimiento y, finalmente, escasa preparación previa de la organización receptora.

Palabras clave: transferencia de tecnología, innovación tecnológica, modelo 1 a 1, organización escolar, tecnología educativa.

\section{SUMMARY}

We start from the technology transfer model to analyse how schools receive their activities and incorporate technological equipment. Particularly, we analyse the innovations generated by these processes at the organizational level of schools as recipients of technology. The studied data are broken down in an investigation currently being performed in 12 regions on the School 2.0 program. At this stage we handle descriptive statistics and references of some of the interviews and classroom observations conducted in the participating centres on the "smart school". From the first analysis, we are able to say that in the case of the school system, it follows a particular transfer pattern: high dependence on the political level, there is more emphasis on the instrumental equipment in knowledge and, finally, little advance preparation of the recipient organization.

Key words: technology transfer, technological innovation, model 1 to 1 , school organization, educational technology.

\section{SOMMAIRE}

Nous commençons à partir du modèle de transfert de technologie pour analyser la façon dont les écoles reçoivent leurs activités et d'intégrer l'équipement technologique. Particulièrement regarder les innovations générées par ces processus au niveau organisationnel des écoles en tant que bénéficiaires de la technologie. Les analysées données sont décomposées dans une enquête actuellement menée dans 12 régions sur le programme de l'école 2.0. A ce stade, nous traitons des statistiques descriptives et des références à certaines des entrevues et des observations en classe menées dans les centres participants à l'école intelligente. Après les premières analysées, nous pouvons dire que dans le cas du système scolaire suit un transfert de motif particulier: la forte dépendance sur le plan politique, il est davantage l'accent sur l'équipement instrumental dans la connaissance et, en définitive, peu de préparation du organisme bénéficiaire.

Mots clés: le transfert de technologie, l'innovation technologique, le modèle 1 à 1, l'organisation scolaire, technologie éducative.

\section{INTRODUCCIÓN}

Partimos del supuesto según el cual el equipamiento tecnológico llega a los centros conforme a un protocolo, conocido como transferencia de tecnología 
(TT en lo sucesivo), ya muy sistematizado en unos ámbitos organizacionales y no tanto en otros. En la sociedad actual este proceso se considera estratégico, por cuanto los sistemas de innovación y desarrollo producen inventos que luego se han de transferir a las diferentes instancias y organizaciones, siendo la escolar una de éstas. La expresión TT alude al "proceso de transmisión del saber hacer (knowhow, savoir faire), de conocimientos científicos y/o tecnológicos y de tecnología de una organización a otra" (Jiménez, 2003, 1). De modo que este proceso afecta por igual a los artefactos tanto materiales como inmateriales o simbólicos, convirtiendo la transferencia en una especie de conocimiento aplicado en contextos distintos. Ahora bien, ¿las iniciativas de equipamiento de los centros pueden estudiarse desde la perspectiva de la TT? Entendemos que sí, al igual que ya se hace en otras organizaciones, extremos que trataremos de argumentar en lo que sigue.

El foco de atención del estudio se centra en la articulación e implementación de los planes de тT a los centros escolares. A partir de la literatura existente en el campo, aunque escasa en el de educación, sugerimos que el protocolo seguido en la transferencia puede ser determinante, en alto grado, de la variedad y naturaleza del uso que en los centros se haga de los recursos tecnológicos. Ciertamente hay abundantes estudios y publicaciones sobre el equipamiento de los centros, impacto en los aprendizajes o si el profesorado lo utiliza mucho o poco y por qué (Mominó et al., 2008; OECD, 2011; Pedró, 2011; Solá y Murillo, 2011). La mayoría de estos trabajos ponen de manifiesto la débil integración de los equipamientos en las prácticas ordinarias de enseñanza y aprendizaje, lo cual nos invita a indagar en las razones de por qué se produce este hecho, casi exclusivo de los ámbitos escolares. ¿Tendrá algo que ver con esta circunstancia el patrón de TT; esto es, cómo se planifica, selecciona, prepara y evalúa la dotación asignada a las instituciones educativas?

La preocupación por la TT no es nueva, en los años 70 la transferencia se planteaba en términos de "máquinas-herramientas" y ahora se aborda como un fenómeno vinculado a la gestión del conocimiento en organizaciones "ligeras" que aprenden de sus experiencias de mejora (Marshall, 1986). Desde planteamientos multidisciplinares la TT se ha convertido en un complejo proceso de innovación en las organizaciones para que alcancen sus objetivos de modo más eficiente. Sobre la base de estas consideraciones tomamos el proyecto de Centros Educativos Inteligentes, auspiciado por la Generalitat Valenciana, a fin de estudiarlo desde la perspectiva de la $\mathrm{TT}^{1}$. Y lo hacemos con un doble propósito: descubrir cómo en el proyecto que nos ocupa se articula la propuesta y prepara a la organización receptora y, en segundo lugar, cómo perciben y se implican los diferentes actores

1. Estudio derivado del Proyecto EDu2010-17037 (Plan Nacional de I+D+i): Las políticas de un "ordenador por niño" en España. Visiones y prácticas del profesorado ante el programa Escuela 2.0. Un análisis comparado entre comunidades autónomas. Investigador principal: Manuel Area Moreira de la Universidad de La Laguna. 
escolares en tales procesos. En definitiva, ¿qué iniciativas se adoptan respecto a la organización de los centros cuando se les dota de nuevos artefactos Tic? Del proyecto objeto de estudio y de cómo se adopta en los centros será de lo que nos ocupemos en los apartados siguientes.

\section{APROXIMACIÓN AL CONTEXTO DEL PROBLEMA}

La institución escolar, por peculiar que sea su relación con las tecnologías, no permanece ajena a las contingencias de una sociedad constituida en torno a la "economía del conocimiento". De hecho, la consigna aparece con meridiana claridad en un documento programático del Gobierno de España en el cual, siguiendo las pautas de la OCDE y la Agenda de Lisboa, se argumenta que la "economía de la innovación pivota sobre un núcleo central, que es el conocimiento y su transferencia, desde aquellos puntos en los que se genera" (MICINN, 2010, 25) ${ }^{2}$. En este entorno macropolítico, desde luego, la institución escolar queda bastante desprotegida ante las reglas que rigen el mercado de las tecnologías, tal como ponen de manifiesto para el caso francés Bouquillion, Miège y Moeglin (2011, 107 y ss.).

Los programas de transferencia incorporan todo tipo de tecnología generada por cualquiera de los agentes integrados en el ecosistema tecnocientífico. Como ya se ha señalado, lo que se transfiere de una organización a otra es el know-how, pero que, en realidad, este intangible engloba productos tan diferentes como: proyectos de $\mathrm{I}+\mathrm{D}+\mathrm{i}$, equipamiento, servicios, asistencia técnica, producción de bienes protegidos, explotación y desarrollo de patentes, propiedad intelectual, movilidad de personal, etc. La innovación y, por tanto, la TT se producen tanto en el sector industrial como en el de servicios, pudiendo afectar a: productos, procesos, posición del producto o servicio en el contexto y el paradigma, cuando una organización cambia el modelo de producción del bien o servicio que presta, como sería el caso de la mutación en "virtual" de la enseñanza presencial (COTEC, 2011, 117 y ss.).

En los sistemas escolares el fenómeno de la transferencia es antiguo, si bien intensificado ahora con las TIC, del cual podemos destacar dos planos bien distintos: el cómo y qué tecnología se transfiere y, en segundo lugar, la naturaleza de las estrategias mediante las cuales se incorporan las tecnologías transferidas a la organización receptora y, en consecuencia, qué cambios se adopta en este plano. Pues, como señala Castells (2001, 121), el informacionalismo genera "una nueva forma de estructura social" al cambiar los dispositivos organizativos de las relaciones de producción. Ambos aspectos son fundamentales y, por tanto, de ellos nos ocuparemos en los subapartados segundo y tercero, mientras que en el primero

2. En la reciente Ley $14 / 2011$, de 1 de junio, de la Ciencia, la Tecnología y la Innovación (BOE, n. ${ }^{\circ}$ 131, de 2 de junio de 2011), se dedica el Título III a regular el fomento de la innovación y la transferencia de conocimiento y tecnología. 
recogemos algunos datos significativos que motivan la línea de indagación en la que se inscribe este trabajo.

\subsection{Por qué buscar otras vías de exploración}

La TT se "asume como un proceso central para la innovación y el desarrollo", tanto de la organización como de la actividad/producto implicado (Pinto 2012, 43). Sin embargo, en el caso escolar los datos no acaban de confirmar tal supuesto, pues se observa, en términos globales, una cierta desproporción entre el esfuerzo de equipamiento y el grado de uso de éste. En todo caso, su incidencia se detecta en la concepción y reorganización de las prácticas de enseñanza, precisamente sobre lo que no hay demasiados análisis (el cambio de paradigma referido más arriba).

De acuerdo con estudios promovidos desde la OCDE, «el 93\% de los alumnos de 15 años de edad de la OCDE asisten a una escuela en la que cuentan con acceso a un ordenador y prácticamente el mismo porcentaje $(92,6 \%)$ dispone igualmente de acceso a Internet" (Pedró, 2011, 17 y ss.). Pese a todo, conforme a este mismo trabajo, el equipamiento que los alumnos tienen en su entorno familiar es mejor que el que les ofrecen en los centros escolares, con un diferencial de casi 18 puntos porcentuales de media entre todos los países (en España el porcentaje de desfase está ligeramente por debajo).

Otro estudio reciente desvela que el 65\% del profesorado admite utilizar el ordenador/impresora "casi todos los días" (Barquín et al., 2011, 36). Sin embargo, es un porcentaje significativamente inferior (34\%), pese a reconocer mayoritariamente el valor didáctico de estas nuevas herramientas (86\%), el que admite la escasa incidencia de estos usos en el aprendizaje de los estudiantes. El aludido tercio de profesorado escéptico responde a un perfil de docente joven y con buen manejo de estos medios. Bastante más pesimista es la conclusión que a partir de los datos de la OCDE extrae Pedró $(2011,22)$, en el trabajo citado, cuando afirma: "La tecnología no se usa en todas las materias con igual intensidad y, por consiguiente, hay un porcentaje elevado de materias en las cuales el nivel de integración es prácticamente inexistente...".

De modo que los agentes industriales transfieren "productos" tecnológicos a los sistemas escolares, la cuestión es que no parece satisfacer las expectativas de innovación alentadas por quienes los promueven. En la mayoría de los casos sólo se transfieren los equipos, no el conocimiento que arropa la implantación y desarrollo de aquéllos en las organizaciones educativas. Pero la presencia de estos equipos, sobre todo los digitales, no es indiferente a las condiciones materiales, culturales y tecnológicas de las organizaciones (Lam, 2000) o a la imposición de ciertos "totalitarismos cibernéticos" (Lanier, 2011). Sobre algunas de las dimensiones afectadas hay ya importantes análisis, como los trabajos sobre "epistemología digital" (Lankshear y Knobel, 2003), sobre la alfabetización visual y digital (Messaris, 1994; Buckingham, 2008), sobre la percepción del espacio y tiempo que tienen los usuarios de las plataformas de e-learning (García del Dujo et al., 2010), sobre 
la nueva "morfología del trabajo" (Antunes, 2012) o sobre los complejos vericuetos psicosociales que sigue el usuario para "aceptar" o no una determinada tecnología (Schwarz y Chin, 2007).

Desde luego, se trata de tópicos novedosos a integrar en la trama organizativa de los centros, lo cual no es nada fácil por cuanto aquélla se articula conforme a una tecnología de proceso específica, por lo demás como el resto de organizaciones (Alcaide Castro, 1982). Extremo que a través de estudios en otras organizaciones, ya han puesto de manifiesto trabajos como los de Gurrutxaga (2010), Johnson (2011) o los que focalizan su atención sobre cómo regular y gobernar la transferencia (Onken and Fisher, 2005). De manera que parece oportuno detenerse a revisar con detalle en qué medida la TT toma en consideración todas estas peculiaridades de las organizaciones. En este sentido el proyecto de Centros Educativos Inteligentes (CEI en lo sucesivo) resulta relevante para analizarlo en el caso de las organizaciones escolares, entre otras razones porque pone especial énfasis en el centro como totalidad.

\subsection{El "Centro Educativo Inteligente»: alternativa al plan "Escuela 2.0"}

El proyecto CEI es, sin duda, un caso de TT del que es necesario explicitar algunas características de su concepción, y con ello facilitar la interpretación de los datos de campo. Lo primero a destacar es que la Conselleria de Educación del Gobierno Valenciano no se adhiere en 2009 al Programa Escuela 2.03, promovido por el Ministerio de Educación. Aduce para justificar tal decisión, entre otros argumentos, el no haber sido consultada con anterioridad sobre una propuesta con importantes repercusiones económicas y políticas. En particular y respecto a esto último, desde la Dirección General de Innovación Tecnológica se subraya que la iniciativa del Gobierno central resta valor al trabajo realizado en el desarrollo de los proyectos propios tales como: ITACA, Mes-Tic, Recicla'ls, IntegraTic y, muy especialmente, el LliureX ${ }^{4}$ focalizado en la adopción del software libre. Se señala, por otra parte, como argumento crítico el que el plan ministerial requiere infraestructura con capacidad suficiente para soportar los nuevos equipamientos, entre ellos la dotación de un portátil para cada alumno de los últimos cursos de primaria y el primer ciclo de la secundaria. En el curso 2009-2010 eran ya cerca de 100.000 ordenadores instalados en los centros educativos valencianos y la nueva dotación representaba aproximadamente otros 50.000 nuevos ordenadores (se estiman unos

3. En http://www.ite.educacion.es/es/escuela-20 se pueden consultar sus características más relevantes. Justificando el recorte en los Presupuestos Generales de 2012 se afirma: «[...] dada la probada ineficacia del Programa Escuela 2.0 (un ordenador/un niño) en el que se gastaron más de 91 millones de euros en 2011, se sustituye por el Programa TIC 2012 con un dotación de 41,5 millones de euros". Consultado el 4 de abril de 2012. http://www.lamoncloa.gob.es/.

4. En http://liurex.net/home/ Consultado el 15 de noviembre de 2011. 
48.500 alumnos de quinto de primaria en ese mismo curso, en la enseñanza pública y concertada). En apreciación de la Administración autonómica la implantación de la iniciativa ministerial generaba no pocos problemas de articulación y, sobre todo, se desviaba de las directrices adoptadas desde la Conselleria.

De modo que la Generalitat Valenciana propone como plan alternativo al Escuela 2.0, el no menos controvertido proyecto Centro Educativo Inteligente. El ambicioso objetivo del mismo es el de integrar las TIC en la totalidad de espacios constitutivos de los centros escolares. Durante el curso 2009/2010 se pusieron en marcha, como centros piloto, un CAEs de infantil y primaria y un instituto de secundaria. En el curso 2010/2011 se sumaron, a la fase de implementación y última de momento, otros 16 centros educativos tanto de primaria como de secundaria. Cabe advertir, no obstante, que un equipamiento con parecidas características recibirían en el futuro el resto de la red de centros públicos, sólo que en los CEI se pretende dotar de una estructura "inteligente" al conjunto de tecnologías y organización.

En este tipo de centro cada aula ordinaria dispone de un ordenador (cliente ligero) para el profesor como apoyo a su tarea docente, ya que permitirá acceder a recursos educativos digitales (del servidor de centro o a través de Internet), y además permitirá realizar gestiones administrativas y académicas mediante la aplicación ITACA ${ }^{5}$ (pasar lista, retrasos, faltas, incidencias, notas, etc.); una pizarra digital interactiva (PDI en lo sucesivo) que permitirá exponer los contenidos educativos de una forma colectiva y atractiva; además de un proyector y el acceso a Internet.

Aparte de las aulas de informática y las aulas ordinarias, estos centros disponen del rincón multimedia en todas las aulas de infantil (si es de primaria), un servidor multimedia para el aula de música, ordenadores (clientes ligeros) en laboratorios y talleres, en las aulas PASE, en las de diversificación curricular, en las de comunicación y lenguaje y en las de pedagogía terapéutica. También se informatizan las salas del profesorado, el servicio de orientación, los departamentos didácticos y las bibliotecas.

Una de las novedades del CEI es la apuesta por el cliente ligero en la mayoría de los puestos de trabajo, tanto del profesorado como de estudiantes. Es una tecnología que permite: reducir el mantenimiento al no poseer sistema operativo ni ningún otro software instalado, por lo tanto no hay que instalar, actualizar, no hay virus, etc. Por otro lado, permite ahorrar en consumo eléctrico hasta un 80\%, reducir la generación de calor y ruido, además de reducir la necesidad de reposición, dado que estos terminales no necesitan ser renovados cada 4 o 5 años como los ordenadores, operación que únicamente se ha de realizar con los servidores de centro y aulas de informática para soportar software actualizado que necesita más capacidad de procesamiento.

5. Acrónimo que significa: Innovación Tecnológica Administrativa para Centros y Alumnos y que sucede a la aplicación Gestión de Centros (GESCEN). 
Otra novedad es la dotación de aulas móviles, compuestas por un equipamiento de 35 portátiles en secundaria, 25 en primaria y acceso a Internet, con el objetivo de que todo el alumnado pueda interactuar con las TIC desde sus pupitres; además de las aulas de informática tradicionales. La siguiente apuesta es el modelo de centro LliureX, cuya principal característica es el servidor de centro que opera la gestión de usuarios. Este servidor centraliza y distribuye los servicios y funcionalidades de todos los ordenadores del centro escolar, incluyendo tanto las aulas de informática como el resto de puestos de trabajo informáticos en cualquier dependencia del centro. Al aumentar la interoperabilidad se simplifica todavía más la gestión de las aulas, ya que basta con realizar la gestión de usuarios, contraseñas y ficheros en el ordenador principal, distribuyéndose automáticamente hasta los servidores de aula. Ahora bien, a nadie se le escapa que la operatividad del proyecto depende de la conectividad de los centros, detalle en el que parece que no se ha reparado lo suficiente, pues en un reciente informe ya se llama la atención sobre la mala conectividad de la mayoría de los centros públicos valencianos (Paniagua Fuentes, 2010, 115 y ss.).

\subsection{El proyecto CEI desde la perspectiva de la TT}

Como se desprende del apartado precedente, el proyecto CEI es producto, en buena medida, del tenso juego de poder entre la Administración central y la autonómica, mediante el cual además de facilitar medios se implica a distintos agentes. Si tomamos el modelo de Rubiralta Alcañiz (2004, 17 y ss.) para el análisis de la TT, observamos que la estructura sobre la que se sustenta el proyecto CEI es la Conselleria d'Educació de la Generalitat Valenciana que, a través de la Direcció General d'Innovació Tecnológica, coordina la implantación en su entorno inmediato del proyecto citado a través del modelo de "Plan piloto". La estructura se fortalece al contar con SMART Board como socio tecnológico, multinacional que comercializa las PDi con mayor implantación en estos centros, además de otras empresas "nodriza" pequeñas o filiales de corporaciones del sector. Por lo que se refiere al equipamiento de los ordenadores "cliente ligero" el proveedor es Inves, fabricante de los mismos y perteneciente al Grupo El Corte Inglés.

En segundo lugar, la estructura se consolida, según Rubiralta, activando instrumentos tales como contratos, cesión de licencias, movilidad del personal, servicios de apoyo, etc. En el caso que nos ocupa se dota a los centros que participan en el proyecto con ordenadores clientes, ordenadores portátiles, servidores de aula y de centro, pizarras digitales. Dotación que se acompaña de software educativo, material didáctico, formación del profesorado, redes de intercambio de experiencias, licencias de usuarios y servicios de mantenimiento, etc. ${ }^{6}$. Los centros del proyecto

6. Las ayudas y materiales de carácter pedagógico pueden encontrarse en: http://mestreacasa. gva.es/web/guest/inicio y en http://www.smarttech.com/es/. 
cuentan con el apoyo, además de la coordinadora o coordinador Tic perteneciente al claustro, con los técnicos del servicio de Soporte y Asistencia Informática (SAI) ${ }^{7}$, el equipo técnico de apoyo al programa y varias empresas que prestan un servicio especializado a los centros más alejados de las capitales. Al igual que otros Gobiernos autónomos, la Generalitat ha generalizado en la red de centros el uso de software libre (Lliurex), que ha planteado no pocos problemas de operatividad, especialmente en el caso de los ciclos formativos.

El tercer elemento del modelo de Rubiralta es la participación o implicación de los diferentes actores. Por la naturaleza de las organizaciones que nos ocupan, ésta es una dimensión fundamental, en la medida que se externalizan ciertas tareas y en su ejecución se implica a nuevos actores no siempre relacionados con la educación ni vinculados al centro. Todo lo cual configura un complejo entramado de relaciones con nodos críticos respecto a la eficiencia. Los proyectos en tales circunstancias responderían al patrón del "modelo mediterráneo" de TT según Rubiralta (los otros modelos son el nórdico, el anglosajón y el centroeuropeo). En definitiva y siguiendo a Aceytuno y Cáceres (2011, 11 y ss.), el proyecto CEI se configura como subsistema local (se descuelga del ministerial y se circunscribe a 18 centros de la Comunidad Valenciana), aprovecha sinergias en ocasiones informales (de los ayuntamientos, por ejemplo), y su fuerte en la transferencia no son tanto las patentes que genera como la proliferación de publicaciones, estudios, congresos, reuniones de carácter científico y formativo ${ }^{8}$.

\section{CONSIDERACIONES DE ORDEN METODOLÓGICO}

Tal como ya se ha mencionado el foco de análisis lo constituye el proyecto de TT denominado CEI, y que tratamos aquí como un caso de estudio a abordar desde el enfoque evaluativo (Stake, 2010). El objetivo general, del que este trabajo es una parte, no es otro que el de explorar claves explicativas del insatisfactorio rendimiento de los medios transferidos, por cuanto no satisfacen las expectativas respecto a la mejora e innovación en las prácticas de enseñanza y aprendizaje en las instituciones escolares. Ahora bien, en el apartado precedente tratamos descriptivamente la concepción y gestión del despliegue dado al citado proyecto, por lo que el paso siguiente es abordar cómo ha sido su recepción en las organizaciones escolares. Tarea que acometemos recopilando distintos datos de campo bajo la orientación de los siguientes objetivos particulares:

a) Reconstruir el perfil de equipamiento y tipología del uso didáctico de los medios transferidos.

7. En http://sai.edu.gva.es/ Consultado el 21 de enero de 2012.

8. Respecto a las diferentes ediciones de congresos relacionados con el programa puede cotejarse en: http://congreso.lliurex.net/index_va.php. 
b) Identificar el patrón que condiciona el grado de aceptación del «modelo CEI" por parte del profesorado.

c) Describir los elementos característicos de la gestión seguida por la Administración educativa en relación al proyecto.

La muestra objeto de estudio la forman los 18 centros de primaria y secundaria que participan en el proyecto CEI de la Comunidad Valenciana (CV en lo sucesivo). Como ya se ha apuntado, los datos que manejamos se desagregan de los obtenidos en el estudio general realizado en los centros de 12 comunidades autónomas (10 participaban en el plan Escuela 2.0, mientras que la comunidad de Madrid y Valencia habían diseñado uno propio). El cuestionario fue elaborado por el equipo de investigación, siguiendo el protocolo habitual para estos casos. Tras ser testeado la versión definitiva se alojó en el espacio web cedido a tal propósito por el ITE (Ministerio de Educación). A la aplicación sólo se podía acceder con una clave específica para cada territorio que, en el caso de la Comunidad Valenciana, se facilitó a los CeI a través de la Direcció General d'Innovació Tecnològica que además les invitaba a colaborar en su cumplimentación. El período para contestarlo se estableció para toda la muestra entre los meses de abril y mayo de 2011, transcurrido este período se cerró el acceso.

A partir de este momento, el ITE entregó el conjunto de los datos al responsable del proyecto para su tratamiento estadístico. Como es lógico, la cumplimentación del cuestionario era anónima y discrecional de los miembros de los diferentes claustros, aun así se recogieron 4.421 cuestionarios válidos, de los que 193 corresponden a la CV. Luego los datos han sido tratados en conjunto y segmentados por comunidades, para lo cual se ha utilizado el programa SPss.18 win?. Herramienta con la que se han efectuado los análisis preliminares de los datos (frecuencias, medias, porcentajes, etc.), así como la fiabilidad y consistencia interna del cuestionario aplicando el coeficiente alpha de Cronbach (Bisquerra, 2000). Por otra parte, desde mayo de 2011 a febrero de 2012, se han realizado entrevistas semiestructuradas a miembros de los equipos directivos, coordinadores TIC y profesorado, además de tres grupos de discusión con estudiantes de segundo de ESO. El material fue grabado y transcrito para buscar en este material, mediante la herramienta ATLAS-TI.7, las categorías que saturan construyendo un modelo explicativo (Trinidad et al., 2006).

Sobre esta base metodológica exponemos y comentamos, en lo que sigue, los primeros resultados relativos al proyecto CEI valenciano mediante estadígrafos descriptivos, al igual que de las entrevistas sólo tomamos las transcripciones. Insistimos en el carácter provisional, sobre todo, porque en la actual fase del trabajo

9. Datos e informes de progreso del proyecto de investigación sobre el programa Escuela 2.0, pueden consultarse en: http://www.edullab.ull.es/ticse20/. 
de observación de campo en cuatro centros nos está permitiendo recopilar nuevas evidencias que enriquecen el significado de estas primeras constataciones.

\section{EXPOSICIÓN Y COMENTARIO DE RESULTADOS}

Desagregados los datos, aclaramos que la muestra autonómica la constituyen 193 cuestionarios válidos de profesoras y profesores. Cabe destacar además de la muestra: se han cumplimentado cuestionarios en los 18 centros que participan en el proyecto valenciano, de los cuales 11 son de infantil y primaria (entre CEIP, CRA y CAES) y 7 institutos de secundaria, algunos de éstos con Ciclos Formativos de FP. La muestra de profesorado que ha contestado se sitúa en los intervalos intermedios, tanto de edad como de experiencia profesional, el porcentaje de profesorado de primaria es ligeramente superior al de secundaria. A partir de los datos aportados por el cuestionario y a la luz de lo expuesto en los epígrafes 2.2 y 2.3, tratamos de interpretar tanto el tipo de tecnología que se transfiere, incluso comparando los datos con el resto de comunidades, como las estrategias organizativas y de apoyo con las que se acompaña la implantación del proyecto.

\subsection{A propósito del equipamiento y uso de las TIC en las aulas}

El perfil que dibuja la Figura 1 a propósito del equipamiento de las aulas de los centros CEI no es demasiado halagüeño, al menos según la apreciación de quienes contestaron a la encuesta. De ésta se desprende que el $24,4 \%$ de las aulas no dispone de Internet ni de ordenador para el profesorado, porcentaje que en la muestra general se reduce al 11,4\% y al 11,8\% respectivamente. Por lo que se refiere al equipamiento de PDI, el 26,9\% dice no disponer de ella y un 38,3\% de la muestra admite no disponer de cañón, una tecnología menos avanzada que la PDI; mientras que en la muestra general no disponen de PDI el 22\% y de cañón el 42,4\%. De manera que los 18 centros de la CV de donde provienen las encuestas se encuentran 5 puntos porcentuales por debajo en la tecnología más avanzada y algo más de 3 por encima en cuanto al cañón de proyección. Retraso de los CEI respecto a la muestra general que se mantiene también en cuanto a la disponibilidad en las aulas de impresoras y, sobre todo, en cuanto al equipamiento de tecnologías más avanzadas como son los Tablets o los iPads donde la diferencia es de casi 6 puntos.

Ahora bien, con el material tecnológico disponible en los centros y en las aulas, ¿en qué y cómo lo utiliza el profesorado? Las tres tareas que reconocen realizar con mayor intensidad es explicar en clase con el apoyo de la PDI (61,1\%), el segundo lugar lo ocupa la realización por parte de los alumnos de ejercicios on-line $(53,9 \%)$ y la tercera es cuando le piden al alumnado búsqueda de información a través de Internet (39,4\%). Mientras que, en la muestra general de la investigación, los porcentajes alcanzados en estas tareas y siguiendo el mismo orden son: $66,9 \%$ explicar con PDI, 67,5\% manda ejercicios on-line y búsquedas un 81,7\%. 


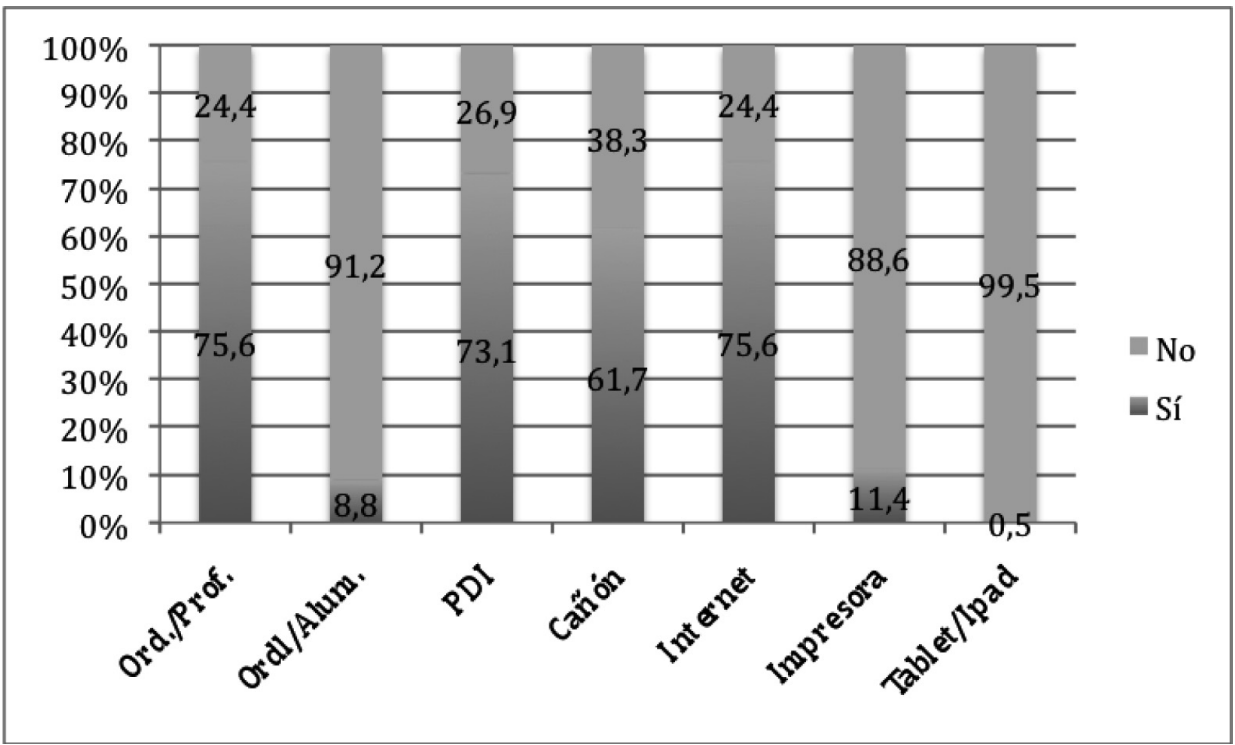

Pese a las muchas diferencias de partida entre el proyecto CEI y el Escuela 2.0, a la postre ambos no han logrado ir mucho más allá de la dotación de tecnologías. Herramientas que en algunos casos de la cV se reconoce usar menos que sus colegas de otras comunidades autónomas. Por ejemplo, mientras que en la muestra general el 69,6\% del profesorado solicita a los alumnos trabajos con procesadores de texto, en el caso de los CEI, dicho porcentaje se reduce al 36,3\%. Por lo que se refiere a las pequeñas producciones multimedia, el 10,4\% de los encuestados valencianos reconocen tenerlos incorporados a su repertorio didáctico, frente al $26,4 \%$ de la muestra general. De modo que estos primeros datos no dejan en buena posición la estrategia de TT activada por el proyecto CEI. Se transfieren herramientas, pero luego no se consigue implicar a los actores en su uso o se hace en un grado cuanto menos insatisfactorio.

\subsection{El modelo CEI visto por el profesorado}

Al margen de otras consideraciones, por ejemplo lo poco afortunado del nombre dado al programa, mantenemos como hipótesis de trabajo en coherencia con el modelo de TT que una de las fortalezas más consistentes de la propuesta política de la CV es focalizar el programa CEI sobre el centro escolar en su conjunto. En última instancia, tanto el equipamiento tecnológico como la diversidad e intensidad de las tareas realizadas van a depender, en alto grado, de las distintas instancias 
organizativas de los centros. Ahora bien, ¿cómo se percibe la dotación asignada y qué estrategias de implementación se han previsto?

\subsubsection{Repercusión del proyecto sobre el centro}

Tal vez el fenómeno más complejo de comprender y gestionar lo encontramos en cómo las estructuras y procesos organizacionales absorben la impronta en los modos de operar de las tecnologías digitales. Entre otras razones porque cada organización tiene la suya propia y ello alienta las resistencias a las nuevas que llegan de fuera limitando su potencial innovador (Mominó et al., 2008). De momento, según hemos podido observar, conviven pautas de organización del trabajo típicas del fordismo junto a otras propiamente postfordistas. Cuestiones que de algún modo subyacen en las respuestas que los encuestados dan al ítem 16 respecto a las diferentes dimensiones de la organización y que recogemos en la figura siguiente.

Figura 2. VAloración del impacto del PROGRAma sobre el CENTRO (ÍTEM 16)

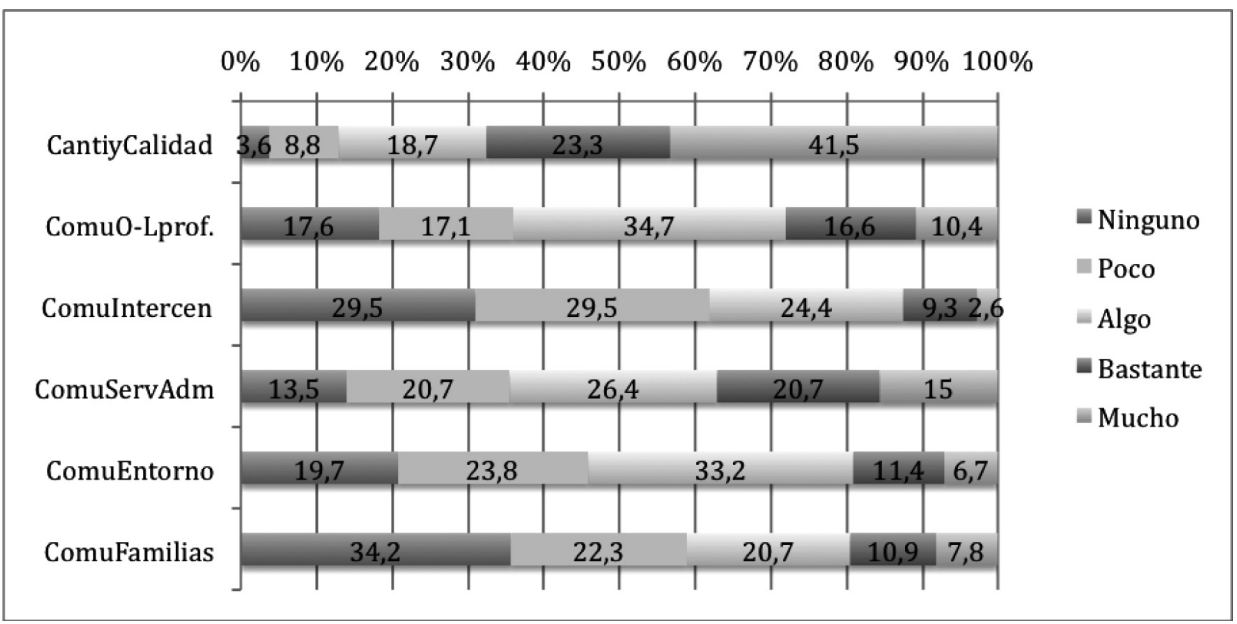

Al igual que reconoce la muestra estatal, la de la CV admite en un alto porcentaje que la mayor contribución del programa CEI se cifra en «mejorar la cantidad y calidad de las TIC en el centro". Por otro lado, algo más de un tercio admite que el programa no ha contribuido nada a incrementar "la comunicación entre profesorado y las familias". Cerca del $60 \%$ de los encuestados reconoce que dicho programa ha contribuido poco o nada a aumentar "el contacto y comunicación con otros centros educativos a través de Internet". La "comunicación on-line entre el 
profesorado del centro" y la comunicación "del centro con otras instituciones" del entorno social, sólo un tercio respectivamente reconoce que ha mejorado "algo".

Pero si no se detecta "ganancia" en lo que respecta a las prestaciones específicas de las tecnologías más avanzadas, ¿qué pasa en su relación con la Administración? Como se desprende de la Figura 2, sólo un 15\% valora que se ha mejorado "mucho" la comunicación de los docentes con las diferentes instancias de la Administración educativa. Mientras que un 34,2\% considera que la mejoría en este plano es "ninguna" o "poca". El director de un CEI de primaria argumentaba: "Qué duda cabe que antes con el GESCEN y ahora con el ITACA, aunque sigue dando algunos problemas, nos facilita mucho las tareas burocráticas... (se sonríe). Bueno, así también nos controlan más desde Conselleria... En fin, donde no veo tan clara su utilización es en las aulas..." (ED-4).

El patrón de respuesta seguido por la muestra de la cV es muy parecido, incluso en porcentajes, al manifestado por el total de la muestra del estudio. Lo que más llama la atención del profesorado es la mejora del equipamiento, sin que luego el potencial de estos medios se despliegue en parecido grado al quehacer de estos docentes. Desde luego, sorprende que aquello que nosotros formulábamos como hipótesis resulte rebatido por los datos. Los nuevos equipamientos, según aprecia el profesorado encuestado, no contribuyen a mejorar lo que es casi constitutivo de las organizaciones, incluidas las escolares. Lo cual obliga a preguntarse por los criterios de selección de los 18 centros "experimentales", así como por la preparación previa del profesorado. Un miembro de la junta directiva de la asociación de directores de institutos de secundaria (ADIES-PV) manifestaba en la entrevista: "Ni en la asociación ni como director de instituto he podido saber cómo se han seleccionado esos 18 centros CEI... Supongo que algo podríamos haber aportado al respecto..." (GAD-1).

\subsubsection{La función de la coordinación TIC}

Este aparente desajuste detectado ¿podría tener que ver con la falta de apoyos para ejercer las nuevas tareas requeridas por el equipamiento recibido? En tal sentido cabe preguntarse por las funciones que desempeña el coordinador/a Tic en los $\operatorname{centros}^{10}$. Cuestiones sobre las que la figura siguiente aporta alguna luz.

Por lo pronto, la práctica totalidad del profesorado reconoce contar con la figura del coordinador TIC en su centro. De hecho, el director de un instituto nos decía: "Antes de pedirle a Conselleria ser CEI me aseguré que el profesor X aceptara ser el coordinador Tic. [...]. Está tan entusiasmado que sin él todo esto no sería

10. La normativa legal de la Comunidad Valenciana (Resolución de 29 de junio de 2010, DOCV 6307, de 09/07/2010) contempla esta figura de coordinación, su designación y el horario de dedicación, pero no sus funciones. 
posible» (ED-1). De todos modos su figura y funciones tienen muchas aristas que aún no se han resuelto o que resultan controvertidas organizativamente (Peirats, Sales y San Martín, 2007). En cualquier caso, según los datos de la figura precedente, las tres funciones que señala en torno a la mitad de los encuestados son las siguientes: "actualizar y administrar los recursos y herramientas informáticas" (74,6\%), ayudar "al profesorado en el desarrollo de la docencia con Tic" (69,9\%) y, en tercer lugar, "organizar la formación del profesorado sobre TIC en el centro" $(67,9 \%)$.

Figura 3. FunCiOnes de la COORDinación (ÍTEM 17)

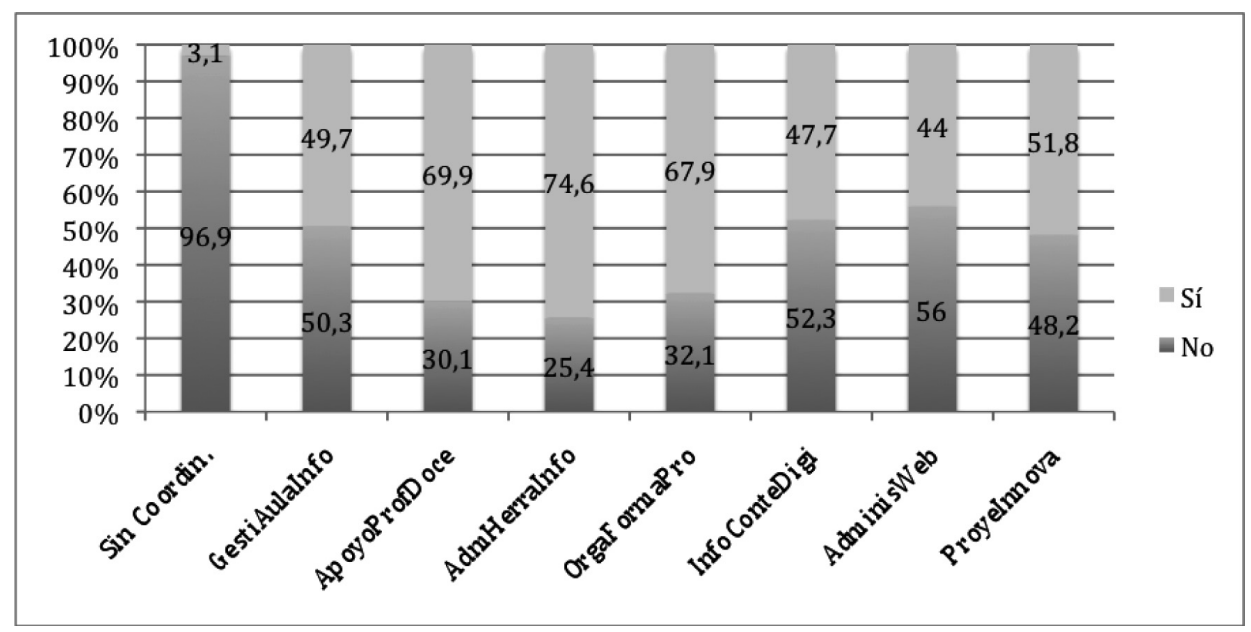

Llama la atención que entre las funciones de la coordinación se destaque, en primer lugar, la instrumental, pero a no mucha distancia se señalan dos de orientación claramente pedagógica, en sentido amplio. De modo que, en el fondo, los encuestados asumen el papel "didáctico" de las TIC y consecuentemente valoran el apoyo que desde la coordinación se les brinda en ese sentido, tal como reflejan las respuestas dadas al ítem 18.

Respuestas que ponen de manifiesto el alto consenso entre los encuestados en torno a la relevancia de la función de coordinación en los CEI. Nada menos que el 89, $1 \%$ considera "muy necesaria" o "bastante necesaria" su presencia para "impulsar y mejorar el uso de las tecnologías". Qué duda cabe que la tecnología cada vez es más amigable pero más compleja su utilización, de ahí que el apoyo de expertos se vuelve también más necesario de cara a optimizar las prestaciones de tales equipos. Supone, en definitiva, integrar en la plantilla docente de los centros a un nuevo especialista, valorado más por el dominio de las nuevas herramientas que por el de los contenidos curriculares e incluso de los metodológicos. Ahora bien, parece interesante seguir indagando sobre cómo las instancias colegiadas 
y el propio docente asumen e integran las nuevas funciones laborales asignadas a la coordinación, qué grado de responsabilidad tiene en la realización de prácticas y proyectos innovadores. Pues como concluyen Hernández Rivero et al. (2011, 324) la figura de la coordinación es crucial ya que se implica en "acciones a nivel de la organización, del desarrollo profesional del profesorado y del diseño del currículo...".

\subsection{A propósito de la politica de implementación de los CEI}

Tal como se puede observar en la Figura 4, casi las tres cuartas partes del profesorado encuestado manifiesta estar "bastante" o "muy de acuerdo" con que la modernización de la educación requiere dotaciones amplias de tecnología en las aulas. Sin embargo, se muestran algo más reticentes a que se haga extensiva la dotación de un ordenador a todo el estudiantado, tan sólo un 31\% estaría "muy de acuerdo" con esta medida. Es posible, no obstante, que los encuestados manifiesten de este modo su desacuerdo con la decisión de la Administración valenciana de no apostar por el modelo 1 a 1 . De hecho, a continuación reconocen no estar demasiado de acuerdo con la política seguida al respecto en la CV (un 38,8\% está nada o poco de acuerdo). ¿Cómo es que asociando la modernización con el equipamiento luego se retraen respecto al plan que dota de un ordenador a cada estudiante? ¿Tal vez sea debido a que no se ha preparado lo suficiente a los actores de la organización receptora, condición necesaria en la TT?

Figura 4. VALORACión De la política TiC EN La CV (ÍTEM 27)

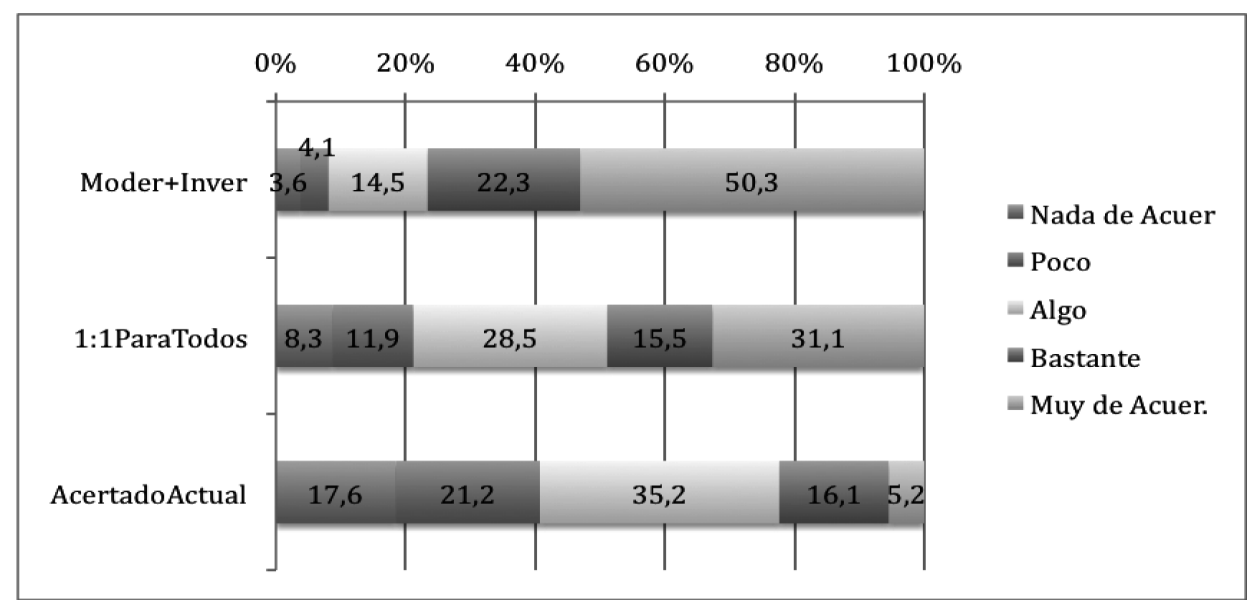


Las organizaciones son "sistemas de interpretación y aprendizaje", concepción desde la que superar la "visión determinista" de la tecnología, máxime cuando los actores interaccionan con las fuerzas tecnológicas del entorno (Lam, 2000). De modo que el proceso de TT debe dar oportunidad a que los actores implicados reflexionen y atribuyan significado a lo que se les propone, a la tecnología que se les proporciona. Sin embargo, en el caso que nos ocupa, no parece que se les haya dado esta oportunidad. Al menos esto es lo que se desprende de las palabras de un coordinador TIC: "La única explicación que encuentro para no participar en el programa del Ministerio es el pique que tienen la administración de aquí y la central. Al final están haciendo lo mismo, llenar de ordenadores los institutos, pero sin la ayuda económica de Madrid y sin saber muy bien para qué..." (EC-2).

Figura 5. VALORACión DE LA IMPLANTACiÓN DEL CEI (ÍTEM 30)

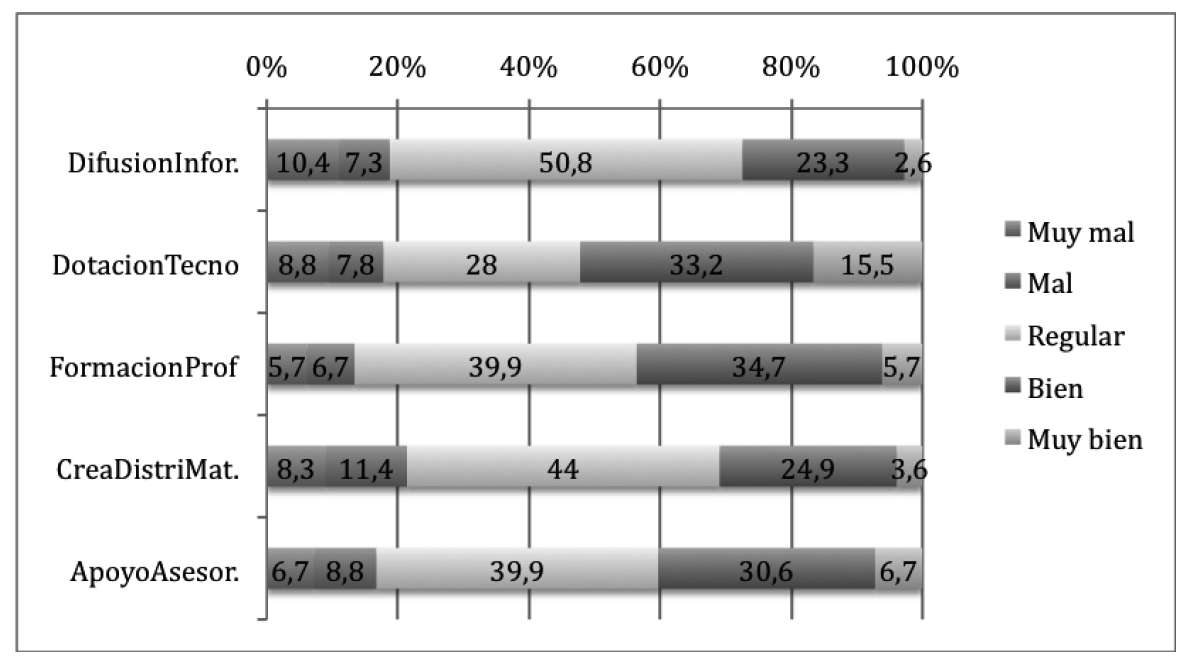

Los datos ponen de manifiesto que la mayoría de los encuestados califica como "regular" O "bien" las cinco dimensiones asociadas a la implantación del programa CEI. Destaca, en coherencia con apreciaciones precedentes, el que un 15,5\% valore como "muy buena" la dotación de equipamiento a los centros. No es que el porcentaje sea demasiado significativo, pero es que en las otras cuatro dimensiones son muy pocos quienes les conceden la máxima valoración. Patrón de respuesta que, por lo demás, es muy semejante al que recoge la valoración de la muestra estatal encuestada. De modo que no estamos ante un fenómeno específico del programa de la $\mathrm{CV}$, por lo cual cabe preguntarse por qué se da esta especie de disociación.

Todo parece indicar que se produce una especie de brecha entre las instancias que diseñan y aplican estos programas "insignia" y las instancias receptoras: los centros escolares (prevalencia del modelo de TT del centro hacia la periferia). Es como 
si los actores captaran con mayor nitidez lo tangible de tales programas (dotación de ordenadores, PDI y demás), mientras que lo intangible que los acompaña pasara más desapercibido; esto es, la gestión que se hace desde las instancias administrativas para su implementación. La falta de coordinación aparece con frecuencia en las entrevistas, así, por ejemplo, el coordinador TIC de un CEI se pregunta: “¿Cómo nos paran desde Conselleria el servidor sin avisar previamente? ¡No se dan cuenta que nosotros también tenemos nuestra marcha de clases, gestiones de secretaría...!" (EC-2). Circunstancias que, a nuestro entender, ponen de manifiesto las debilidades del modelo en el que se sustenta la TT al sistema escolar, aspecto al que convendrá prestar atención en las visitas a los centros y reflexionar con detenimiento, si es que la voracidad mercantil lo permite. En este sentido nos ha llamado la atención en las visitas, aunque las respuestas al ítem 30 no lo reflejen con nitidez, cómo en estos centros se rompe el monopolio del libro de texto impreso en favor de diferentes soluciones en soporte digital ofertadas por las editoriales.

\section{CONCLUSIONES PROVISIONALES}

Como señala Wolcott (2003, 133 y ss.), los trabajos de esta naturaleza no necesitan "conclusiones finales" con las que clausurar el análisis, pues el trabajo interpretativo nunca se puede dar por cerrado. Antes bien, los párrafos finales deben recuperar ideas y reflexiones sobre los asuntos abordados que más hayan llamado la atención. Planteado todo ello en términos que tanto a los lectores como a quien lo redacta les susciten nuevos interrogantes y vías novedosas para seguir indagando sobre un objeto de estudio con tantas aristas como el que nos ocupa. Según nos llama la atención Touraine $(2002,32)$, la instrumentalidad de las tecnologías actuales contribuye a construir un sujeto cuyas referencias emanan del potencial de esa relación productiva y no tanto de la sociedad en la que vive. Por otra parte, como ya se ha indicado, estamos todavía visitando los CEI para hablar con los diferentes actores y observar las prácticas de aula en busca de indicios sobre estas cuestiones.

Es muy pronto todavía para valorar el potencial explicativo del "modelo de TT" respecto a lo que pasa en los centros con la dotación de tecnologías. Ahora bien, sí nos resulta una herramienta muy útil para diseccionar ámbitos relevantes en el diseño e implementación de proyectos como el CEI, pese a no haber sido concebido propiamente como TT. Contamos así con una ayuda importante para avanzar en la comprensión del complejo proceso de asimilación de las tecnologías digitales en los centros, abriendo nuevas vías exploratorias en las distintas fases de despliegue del proyecto en su estructura, instrumentos e implicación, tal como señalan Rubiralta (2004), Schwarz y Chin (2007) o Rodríguez Pomeda (2007). Constatación que nos permite considerar consistente el objetivo principal que inspira este trabajo y, en todo caso, sobre el que se ha de seguir indagando una vez concluida la recogida de datos de campo. Tarea para la que es pertinente considerar los hallazgos vinculados a los objetivos más concretos de este trabajo. 
a) De acuerdo con el primero de ellos y pese a que la TT se agota prácticamente en las iniciativas de dotación de equipos a los centros, el perfil que éstos presentan es medio-bajo, al menos respecto a la dotación disponible en los centros de las otras 11 comunidades autónomas. Diferencia que puede explicarse por el retraso en la recepción de los equipos en los CEI, ya que dicho proyecto se activa con posterioridad al Escuela 2.0, pero también hay una razón técnica: mientras en un caso se limita a ordenadores portátiles y a las PDI en las aulas, en el caso valenciano la "inteligencia" requería complejas y costosas instalaciones en el conjunto del centro que exigían la intervención de técnicos de la conselleria y de empresas externas. Todo lo cual retrasó un tiempo la puesta en marcha de los equipos que los centros tenían asignados. Por otro lado, tal como apuntan los datos, coincidentes con la mayoría de los estudios, el tipo de actividad realizada con estos medios es tendencialmente la de apoyar la explicación de clase, buscar información o realizar ejercicios de clase y no fomenta en demasía la comunicación interna y externa como es propio de estos medios. Desde la perspectiva didáctica cabe preguntarse cómo romper esta tendencia y ampliar el repertorio de uso creativo de estos poderosos medios.

b) Respecto al segundo objetivo específico llama la atención la tibia aceptación del proyecto CEI entre el profesorado, coincidente por lo demás con una de las conclusiones del estudio de Sola y Murillo $(2011,52)$. La percepción que se tiene desde dentro es que ni las prácticas ni los resultados cambian demasiado, salvo significativas excepciones vinculadas a las profesoras y profesores más inquietos de los claustros. Estos docentes, por lo general, suelen ser además quienes ejercen la "Coordinación TIC", figura que suscita gran aceptación entre la mayoría de los colegas encuestados. Por otra parte, como ya se ha señalado en relación al sistema escolar, la TT sólo afecta a los artefactos y no al conocimiento en el que se fundan tales innovaciones. Se sabe que las organizaciones se proveen de tecnologías propias para el logro de sus objetivos, tecnologías no del todo compatibles con las promovidas por los equipos transferidos. No sólo se trastoca el espacio, el tiempo y los protocolos de actuación, sino también los de control, autoridad, autonomía, ámbitos de responsabilidad, estructura de las relaciones laborales, dispositivos epistémicos sobre el conocimiento, etc.

c) El tercer y último objetivo hace referencia a las características de la gestión por parte de la Administración de este tipo de proyectos y que consideramos un factor clave. Más de un tercio del profesorado encuestado no se siente interpelado por la iniciativa CEI, al considerar que se concibe y decide desde arriba, como asunto "político", sin contar demasiado con quienes han de aplicarla. Otros elementos atribuidos a la iniciativa son las escasas estrategias de acompañamiento, pues las pocas que se activan son inconexas y promovidas por instancias de diferente adscripción administrativa e incluso entidades ajenas al ámbito escolar. Hecho que dificulta en alto grado la coordinación de las actuaciones que evitarían el desconcierto entre los agentes del centro, cuando Onken y Fisher $(2005,53)$ consideran la coordinación un factor crítico en la gestión de la transferencia. Y es que, por lo 
que se refiere al sistema escolar, los programas de TT son verdaderos puzles confeccionados con piezas de difícil encaje, que van desde las políticas de dotación improvisadas y normas de uso, hasta la falta de preparación previa de los actores directamente implicados, pasando por la provisionalidad de este tipo de proyectos (en estos momentos el Escuela 2.0 está "desaparecido" y el CEI "congelado").

Para terminar y con carácter general, cabe advertir que el proyecto CEI provoca un cambio de posición social en el servicio que presta, en la medida que estos centros son considerados más "avanzados" que el resto, lo cual les confiere un plus de prestigio en su entorno. Por otro lado, algunos de estos CEI plantean un "cambio de paradigma" en el modelo de enseñanza, en tanto que ya proponen muchas tareas (ejercicios de clase, realización y evaluación de trabajos, comunicación con padres, etc.) en formato on-line. Circunstancia que tiene importantes implicaciones políticas y pedagógicas respecto al carácter público del sistema escolar, así como las condiciones en las que se presta. Lo cual nos obliga prospectivamente a indagar sobre cuestiones tales como: ¿Hasta qué punto estos programas de TT constituyen un paso más en la quiebra del pacto moderno por la escolaridad? ¿Qué principios de economía política rigen estos programas tan imprecisos en lo pedagógico? ¿Cómo se están transfiriendo los contenidos curriculares de los libros de texto convencionales a los soportes digitales?

\section{REFERENCIAS BIBLIOGRÁFICAS}

ACEYTUnO, M. T. y CÁCERES, F. R. (2011) Los modelos europeos de transferencia de tecnología universidad-empresa. Ponencia presentada a la XIII Reunión de Economía Mundial. Consultado el 15 de febrero de 2012. http://xiiirem.ehu.es/es/programa-detallado.

AlCAide CASTRO, M. (1982) Las nuevas formas de organización del trabajo. Madrid, Akal.

ANTUNES, R. (2012) La nueva morfología del trabajo y sus principales tendencias: informalidad, infoproletariado, (in)materialidad y valor. Sociología del Trabajo, 74, 47-68.

Barquín, J. et al. (2011) Los datos de las tic en los centros, en Sola, M. y Murillo, J. F. (coords.) Las TIC en la Educación. Realidad y expectativas. Informe anual 2011. Madrid, Fundación Telefónica.

Bisquerra, R. (2000) Métodos de investigación educativa. Guía práctica. Barcelona, CEAc.

Bouquillion, Ph.; Miège, B. y Moeglin, P. (2011) La situación de la industria creativa. Un debate significativo en Francia, en Bustamante, E. (ed.) Industrias creativas. Amenazas sobre la cultura digital. Barcelona, Gedisa, 97-116.

Buckingham, D. (2008) Youth, identity, and digital media. Cambridge, MIT Press.

CAstells, M. (2001) La galaxia Internet. Madrid, Areté.

COTEC (2011) Informe COTEC 2011. Tecnología e innovación en España. Madrid, COTEC.

García del Dujo, Á. et al. (2010) Análisis del tiempo en los entornos virtuales de formación. Teoría de la Educación, 22 (2), 111-130.

GurrutXaga ABAD, A. (2010) Recorridos por el cambio, la innovación y la incertidumbre. Bilbao, Servicio Editorial de la Universidad del País Vasco.

HERnÁndez Rivero, V. et al. (2011) El coordinador TIC en la escuela: análisis de su papel en procesos de innovación. Profesorado. Revista de Currículum y Formación del Profesorado, 15 (1), 315-327. 
JimÉNEZ, J. M. (2003) Aspectos de la eficiencia en la transferencia de tecnología. Revista de Investigación en Gestión de la Innovación y Tecnología, 14, 15. Consultado el 23 de marzo de 2012. http://www.madrimasd.org/revista/revista14/.

Johnson, S. (2011) Las buenas ideas. Una historia natural de la innovación. Madrid, Ediciones Turner.

LAM, A. (2000) Tacit knowledge, organizational learning, societal institutions: and integrated framework. Organization Studies, 21, 487-513.

Lanier, J. (2011) Personas desaparecidas. Claves de Razón Práctica, 218, 68-74.

Marshall, G. (1986) La moderna tecnología de la información. Madrid, Alhambra.

Messaris, P. (1994) Visual Literacy. Image, Mind and Reality. San Francisco, Westview Press.

MICINN (2010) Estrategia Estatal de Innovación (e2i). Madrid, Ministerio de Ciencia e Innovación. Consultado el 28 de noviembre de 2011. http://www.micinn.es/.

Mominó, J. M. et al. (2008) La escuela en la sociedad red. Internet en la educación primaria y secundaria. Barcelona, Ariel.

OECD (2011) PISA 2009 Results: Students on-line. Digital Technologies and Performance, vol. VI. Consultado el 16 de abril. http://dx.doi.org/10.1787/9789264112995-en.

OnKen, M. y FisheR, C. (2005) Perceived Impacts of Government Regulations on Technology Transfers. Journal of Nonprofit \& Public Sector Marketing, 13 (1/2), 35-55.

Paniagua Fuentes, J. (dir.) (2010) Libro Blanco de la enseñanza secundaria. Comunidad Valenciana 1998-2007. Valencia, UNED-Alzira-Valencia y Generalitat Valenciana.

Pedró, F. (2011) Tecnología y escuela: lo que funciona y por qué. Documento básico. XXVI Semana Monográfica de la Educación. Consultado el 12 de enero de 2012. http://www. fundacionsantillana.com/.

Peirats, J.; Sales, C. y San Martín, A. (2007) Funciones del coordinador en la organización de los programas de introducción informática. El caso de dos escuelas de primaria, en I Congrés Internacional Escola y TIC. Alicante. Consultado el 10 de febrero de 2012. http://www.dgde.ua.es/congresotic/.

PINTO, H. (2012) Instituciones, innovación y transferencia de conocimiento: contribuciones de los estudios sobre las variedades de capitalismo. Arbor. Ciencia, Pensamiento y Cultura, 188 (753), 31-47.

Rodríguez Pomeda, J. (2007) La transferencia de tecnología en España. Diagnóstico y perspectivas. Economía Industrial, 366, 15-22.

Rubiralta AlcañIz, M. (2004) Transferencia a las empresas de la investigación universitaria. Descripción de los modelos europeos. Madrid, Fundación COTEC.

SCHWARZ, A. y ChIN, W. (2007) Looking forward: Toward an Understanding of the Nature and Definition of IT Acceptance. Journal of the Association for Information Systems, 8 (4), 13-21.

Sola, M. y Murillo, J. F. (coords.) (2011) Las TIC en la Educación. Realidad y expectativas. Informe anual 2011. Madrid, Fundación Telefónica.

STAKe, R. E. (2010) Investigación con estudio de casos. Madrid, Morata.

Touraine, A. (2002) La sociedad desestructurada, en VV. AA. Teorías para una nueva sociedad. Madrid, Fundación Marcelino Botín, 17-46.

TRINIDAD, A. et al. (2006) Teoría fundamentada "Grounded Theory". La construcción de la teoría a través del análisis interpretacional. Madrid, CIS.

Wolcotт, H. F. (2003) Mejorar la escritura de la investigación cualitativa. Medellín, Editorial Universidad de Antioquia. 